Article

\title{
Food Additive Titanium Dioxide and Its Fate in Commercial Foods
}

\author{
Ji-Soo Hwang ${ }^{1}$, Jin Yu ${ }^{1}$, Hyoung-Mi Kim ${ }^{2}$, Jae-Min $\mathrm{Oh}^{3}$ and Soo-Jin Choi ${ }^{1, *(D)}$ \\ 1 Division of Applied Food System, Major of Food Science \& Technology, Seoul Women's University, \\ Seoul 01797, Korea \\ 2 Department of Chemistry and Medical Chemistry, College of Science and Technology, Yonsei University, \\ Wonju 26493, Gangwondo, Korea \\ 3 Department of Energy and Materials Engineering, Dongguk University-Seoul, Seoul 04620, Korea \\ * Correspondence: sjchoi@swu.ac.kr; Tel.: +82-2-970-5634; Fax: +82-2-970-5977
}

Received: 30 July 2019; Accepted: 13 August 2019; Published: 16 August 2019

\begin{abstract}
Titanium dioxide $\left(\mathrm{TiO}_{2}\right)$ is one of the most extensively utilized food additives (E171) in the food industry. Along with nanotechnology development, the concern about the presence of nanostructured particles in $\mathrm{E}^{171} \mathrm{TiO}_{2}$ and commercial food products is growing. In the present study, the physicochemical properties of commercially available $\mathrm{E} 171 \mathrm{TiO}_{2}$ particles, including particle size distribution, were investigated, followed by their cytotoxicity and intestinal transport evaluation. The fate determination and quantification of $\mathrm{E} 171 \mathrm{TiO}_{2}$ in commercial foods were carried out based on the analytical procedure developed using simulated foods. The results demonstrated that $\mathrm{TiO}_{2}$ is a material mainly composed of particles larger than $100 \mathrm{~nm}$, but present as an agglomerated or aggregated particle in commercial foods with amounts of less than $1 \%(\mathrm{wt} / \mathrm{wt})$. Titanium dioxide particles generated reactive oxygen species and inhibited long-term colony formation, but the cytotoxicity was not related to particle size distribution or particle type (food- or general-grade). $\mathrm{All}^{\mathrm{TiO}} 2$ particles were mainly transported by microfold (M) cells, but also by intestinal tight junction. These findings will be useful for $\mathrm{TiO}_{2}$ application in the food industry and predicting its potential toxicity.
\end{abstract}

Keywords: titanium dioxide; nanostructure; size distribution; quantification; fate; commercial foods

\section{Introduction}

Titanium dioxide $\left(\mathrm{TiO}_{2}\right)$, naturally occurring oxide of titanium, is widely utilized as a food additive pigment in confectionary, such as chocolates, candies, bakeries, snacks, and chewing gums due to the fact of its brightening and whitening properties. In the European Union (EU), it is referred to as E171, with no maximum level specified [1-3]. The Joint Food and Agriculture Organization (FAO)/Word Health Organization (WHO) Expert Committee on Food Additives did not establish an acceptable daily intake (ADI) of $\mathrm{TiO}_{2}$, because its oral absorption amount is extremely low (maximum of $0.1 \%$ ) $[1,4,5]$. In the United States, food additive $\mathrm{TiO}_{2}$ has been used since 1966, and the Food and Drug Administration (FDA) recommends its concentration, in general, below $1 \%$ by weight of the food [6,7]. Recent rapid developments in nanotechnology have led to the production of nano-sized $\mathrm{TiO}_{2}$, and it is probable that $\mathrm{TiO}_{2}$ nanoparticles (NPs) are imperceptibly used as food additive E171. However, the current specification for food additive $\mathrm{TiO}_{2}$ does not include characterization of particle size distribution. European Commission Recommendation 2011/696/EU defined a nanomaterial as a natural, incidental or manufactured material composed of more than $50 \%$ particles of 1-100 nm in the number-size distribution [8]. Recently, the European Food Safety Authority (EFSA) Journal reported that food additive $\mathrm{E} 171$ primarily consists of micro-sized $\mathrm{TiO}_{2}$ particles, with a nano-sized $(<100 \mathrm{~nm})$ particle less than $3.2 \%$ by mass or $10-39 \%$ by number-size distribution, obtained by scanning electron 
microscopy (SEM)/transmission electron microscopy (TEM) [1]. Yang et al. [9] also demonstrated that $\mathrm{E} 171 \mathrm{TiO}_{2}$ particles had $19-35 \%$ of nano-sized primary particles by number. In the current state, E171 $\mathrm{TiO}_{2}$ seems to not be considered as a nanomaterial according to the EU Recommendation. However, more extended study is required to assume that commercially available food additive E171 contains less than $50 \%$ of nano-sized $\mathrm{TiO}_{2}$ by number-size distribution. Moreover, information about the fate of $\mathrm{TiO}_{2}$ in commercial foods is necessary to be provided.

As concern about the use of NPs in food products grew, research on determination and quantification of food-grade $\mathrm{TiO}_{2}$ in commercial foods has recently increased [10-12]. However, the reliability of the methods used for $\mathrm{TiO}_{2}$ quantification has not been clearly demonstrated. Indeed, separation of $\mathrm{TiO}_{2}$ from complex food matrices and its quantification in the presence of food components are challenging, because diverse organic food matrices can disturb the accuracy and precision for the characterization and quantification of $\mathrm{TiO}_{2}[13,14]$. In particular, NPs are generally known to be covered by environmental matrices, such as biological and food matrices, forming particle-matrix corona, which surely affects particle size distribution, surface characteristics, and fate [15-18]. Hence, it is necessary to establish an appropriate pretreatment method depending on food matrix type to dissolve organic matrices, which is critical for qualitative and quantitative analysis.

Studies have revealed that the oral absorption and bioavailability of $\mathrm{TiO}_{2}$, measured either as particles or as titanium, are extremely low [19-22]. Biokinetic behaviors of $\mathrm{TiO}_{2}$ were found to be independent of particle size [22], but highly affected by the presence of biomatrices [16]. The potential harmful effects of food-grade $\mathrm{TiO}_{2}$ on the intestinal epithelial barrier, intestinal disease, colorectal cancer, and development in offspring rodents have been recently reported [23-26]. Hence, further toxicity studies on food-grade $\mathrm{TiO}_{2}$ are urgently needed. Moreover, answers to the questions of whether food-grade $\mathrm{TiO}_{2}$ is more toxic than general-grade $\mathrm{TiO}_{2}$ and whether or not the former consists of smaller particle sizes than the latter are required.

In the present study, we characterized the physicochemical properties of food-grade $\mathrm{E} 171 \mathrm{TiO}_{2}$ commercially applied to food products. Pretreatment and analytical procedures for determination and quantification of $\mathrm{TiO}_{2}$ in food matrices were established using representative $\mathrm{TiO}_{2}$-containing simulated foods, and its identification was carried out in commercial foods. Finally, the cytotoxicity and intestinal transport mechanism of $\mathrm{E} 171 \mathrm{TiO}_{2}$ were evaluated and compared with those of general-grade bulk and nano $\mathrm{TiO}_{2}$ in human intestinal cells and in vitro 2D and 3D intestinal epithelium models, respectively.

\section{Materials and Methods}

\subsection{Materials}

Five food-grade $\mathrm{E} 171 \mathrm{TiO}_{2}$ products from four different manufacturers were provided as test materials and numbered as T1-T5. General-grade bulk and nano $\mathrm{TiO}_{2}$ were purchased from Sigma-Aldrich (St. Louis, MO, USA) and Alfa Aesar-Johnson Matthey Co. (Karlsruhe, Germany), respectively. Stock solutions $(1 \mathrm{mg} / \mathrm{mL})$ of particles were prepared by dispersion in distilled water (DW) for $30 \mathrm{~min}$ and sonication for $5 \mathrm{~min}$, just prior to experiments. Commercial products containing $\mathrm{TiO}_{2}$ (chewing gum, candy, chocolate, jelly, sauces, and snacks) from international brands were purchased from markets in Seoul, Korea, in 2018.

\subsection{Characterization}

Primary particle size, morphology, and chemical characterization were determined by SEM (JSM-7100F, JEOL, Tokyo, Japan) equipped with energy-dispersive X-ray spectroscopy (EDS; Aztec, Oxford Instruments, Abingdon, UK). Zeta potentials and hydrodynamic radii of particles were measured with Zetasizer Nano System (Malvern Instruments, Worcestershire, UK). The X-ray diffraction (XRD) patterns were measured using an X-ray diffractometer (D2phaser, Bruker AXS Inc., Madison, WI, USA) with Ni-filtered $\mathrm{CuK} \alpha$ radiation. 


\subsection{Size Fractionation}

Fractionation of T4 particles depending on particle size was carried out using sucrose (Sigma-Aldrich) gradient solution. Four sucrose solutions (10, 20, 30, and 50\%) were prepared and $10 \mathrm{~mL}$ of each sucrose solution was stacked in a conical tube $(50 \mathrm{~mL})$ from high to low concentration order. The T4 suspension $(40 \mathrm{mg} / \mathrm{mL})$ was prepared after stirring for $30 \mathrm{~min}$ and sonication for $5 \mathrm{~min}$, and $10 \mathrm{~mL}$ of the suspension was carefully added on the top layer of sucrose gradient solution $(10 \%)$. Fractionation of the particles was performed by centrifugation at $2000 \mathrm{rpm}$ for $40 \mathrm{~min}$. After centrifugation, the fractionated solution was divided into upper, middle, and lower parts, which were further characterized by SEM.

\subsection{Preparation of $\mathrm{TiO}_{2}$-Containing Simulated Foods}

Sugar powder containing $\mathrm{TiO}_{2}$ was prepared by mixing $4.9 \mathrm{~g}$ of grinded sugar (Samyang Co., Seoul, Korea) and $0.1 \mathrm{~g}$ of $\mathrm{TiO}_{2}$ in an agate mortar. Chewing gum containing $\mathrm{TiO}_{2}$ was prepared by adhering $0.01 \mathrm{~g}$ of $\mathrm{TiO}_{2}$ on the surface of $0.5 \mathrm{~g}$ of gum base, followed by surface coating with melted sugar. The contents of $\mathrm{TiO}_{2}$ in simulated foods were adjusted to $2 \%(\mathrm{wt} / \mathrm{wt})$.

\subsection{Pretreatment of Simulated Foods, Commercial Products, and Cell Samples for Characterization and Quantitative Analysis}

Different pretreatment methods were applied depending on food type. One piece of simulated gum or commercial chewing gum in $15 \mathrm{~mL}$ of DW was stirred overnight. Thus, washed gum base in DW was collected and further washed with $5 \mathrm{~mL}$ of acetone (Samchun Pure Chemical Co., Ltd., Pyeongtaek, Gyeonggi-do, Korea) twice and $5 \mathrm{~mL}$ of ethanol (Samchun Pure Chemical Co., Ltd.), and then, all washed solutions were pooled. Finally, $1 \mathrm{~mL}$ of the pooled solution was pre-digested with nitric acid (Samchun Pure Chemical Co., Ltd.) and hydrogen peroxide (Samchun Pure Chemical Co., Ltd.).

The $\mathrm{TiO}_{2}$-containing simulated sugar powder, other commercial foods $(0.2 \mathrm{~g})$, such as candy, chocolate, jelly, sauces, and snacks, and the cell samples were directly subjected to pre-digestion with nitric acid and hydrogen peroxide. Nitric acid $(10 \mathrm{~mL})$ was added to each sample and heated to $180-200{ }^{\circ} \mathrm{C}$ in order to digest organic materials. Hydrogen peroxide $(1 \mathrm{~mL})$ was then added and heating was continued, and this procedure was repeated until the solution was colorless and clear. The pre-digested samples were resuspended in $1 \mathrm{~mL}$ of deionized and distilled water (DDW), and centrifuged at 10,000 rpm for $20 \mathrm{~min}$. Then, the precipitate was washed twice with $2 \mathrm{~mL}$ of ethanol and re-suspended in $20 \mu \mathrm{L}$ of ethanol for SEM-EDS analysis [27].

\subsection{Quantitative Analysis}

Sulfuric acid (10 mL, Samchun Pure Chemical Co., Ltd.) was added to the pre-digested samples and heated at $200{ }^{\circ} \mathrm{C}$ for $2 \mathrm{~h}$ in order to dissolve Ti from $\mathrm{TiO}_{2}$ particles and heated at $380{ }^{\circ} \mathrm{C}$ until the remaining solution was colorless and removed. The final digested and dissolved products were diluted to DDW ( $5 \mathrm{~mL}$ ) and total Ti levels were analyzed by inductively coupled plasma-atomic emission spectroscopy (ICP-AES; JY2000 Ultrace, HORIBA Yvon Longjumeau, France).

\subsection{Cell Culture}

Human intestinal epithelial Caco-2 cells were supplied from the Korean Cell Line Bank (Seoul, Korea). The cells were cultured in minimum essential medium (MEM; Welgene, Gyeongsan, Korea), which contained $10 \%$ heat inactivated fetal bovine serum (FBS), penicillin (100 units $/ \mathrm{mL}$ ), and streptomycin $\left(100 \mu \mathrm{g} / \mathrm{mL}\right.$, Welgene) in a $5 \% \mathrm{CO}_{2}$ incubator at $37^{\circ} \mathrm{C}$.

\subsection{Short-Term Cell Proliferation}

The short-term effect of particles on cell proliferation was evaluated with water-soluble tetrazolium salt-1 (WST-1; Roche, Molecular Biochemicals, Mannheim, Germany). Cells $\left(1 \times 10^{4}\right.$ cells/100 $\mu$ L) were 
incubated with particles for $24 \mathrm{~h}$. Then, WST-1 solution $(10 \mu \mathrm{L})$ was added to each sample. After further incubation for $4 \mathrm{~h}$, absorbance at $440 \mathrm{~nm}$ was measured using a microplate reader (SpectraMax ${ }^{\circledR}$ M3, Molecular Devices). Cells incubated without particles were used as controls.

\subsection{Cell Membrane Damage}

The effect of particles on cell membrane integrity was monitored with a CytoTox 96 Non-Radioactive Cytotoxicity Assay. Cells $\left(6 \times 10^{4} \mathrm{cells} / \mathrm{mL}\right)$ were treated with particles $(5,50$, 250, and $1000 \mu \mathrm{g} / \mathrm{mL}$ ) for $24 \mathrm{~h}$. Then, the cell culture medium was collected, centrifuged, and 50 $\mu \mathrm{L}$ of aliquots were used for analysis. Substrate solution $(50 \mu \mathrm{L})$ was added to each aliquot, and further incubated for $30 \mathrm{~min}$ at room temperature. After addition of stop solution ( $50 \mu \mathrm{L})$ to the reacting solution, the absorbance at $490 \mathrm{~nm}$ was measured using a microplate reader (SpectraMax ${ }^{\circledR}$ M3, Molecular Devices). Cells incubated without particles were used as controls.

\subsection{Colony-Forming Ability}

The long-term effect of particles on colony formation was evaluated by clonogenic assay. The cells $\left(5 \times 10^{2}\right.$ cells $\left./ 2 \mathrm{~mL}\right)$ were incubated with particles $(5,50$, and $250 \mu \mathrm{g} / \mathrm{mL})$ for 14 days. Then, the cells were washed with phosphate buffered saline (PBS; Dongin Biotech Co., Seoul, Republic of Korea), fixed with 90\% methanol (Samchun Pure Chemical Co., Ltd.), and stained with $0.5 \%$ crystal violet solution (Sigma-Aldrich) for $1 \mathrm{~h}$. After washing the cells with DDW and air-dying, colonies consisting of more than 50 cells were counted. Cells incubated without particles were used as controls.

\subsection{Reactive oxygen species (ROS)}

The intracellular ROS generated by particles was monitored with a peroxide-sensitive fluorescent probe, $2^{\prime}, 7^{\prime}$-dichlorofluorescein diacetate $\left(\mathrm{H}_{2} \mathrm{DCFDA}\right.$; Molecular Probes Inc., Eugene, OR, USA). Cells $\left(1 \times 10^{4}\right.$ cells $\left./ 100 \mu \mathrm{L}\right)$ were treated with particles for $24 \mathrm{~h}$, and then, $20 \mu \mathrm{M} \mathrm{H}_{2}$ DCFDA were added and further incubated for $30 \mathrm{~min}$ at $37^{\circ} \mathrm{C}$ in the dark. After washing with PBS, intracellular dichlorofluorescein fluorescence (DCF) was monitored using a fluorescence microplate reader (SpectraMax ${ }^{\circledR}$ M3, Molecular Devices). Excitation and emission wavelengths were set at 485 and $535 \mathrm{~nm}$, respectively.

\subsection{Apoptosis Induction}

The effect of particles on apoptosis induction was evaluated with annexin V-fluorescein isothiocyanate (FITC) and propidium iodide (PI), according to the manufacturer's protocol (Invitrogen, Carlsbad, CA, USA). Cells $\left(1 \times 10^{6}\right.$ cells $)$ were exposed to particles $(1 \mathrm{mg} / \mathrm{mL})$. After incubation for $24 \mathrm{~h}$, the cells were harvested with a scraper, washed with cold PBS, and then resuspended in binding buffer (cell density of $1 \times 10^{6}$ cells/mL). Then, the cells were stained with annexin V-FITC $(5 \mu \mathrm{L})$ and $1 \mu \mathrm{L}$ of PI $(100 \mu \mathrm{g} / \mathrm{mL})$ working solution for $15 \mathrm{~min}$ at room temperature in the dark. After adding annexin-binding buffer $(400 \mu \mathrm{L})$ to samples, the stained cells were finally measured with flow cytometry (Beckman Coulter, Brea, CA, USA) by counting 10,000 cells.

\subsection{Intestinal Transportation}

Human Burkitt's lymphoma Raji B cells, supplied from the Korean Cell Line Bank, were cultured in Roswell Park Memorial Institute (RPMI) 1640 medium (Welgene), which contained FBS $(10 \%)$, non-essential amino acids (1\%), L-glutamine $(1 \%)$, penicillin (100 units $/ \mathrm{mL})$, and streptomycin $(100 \mu \mathrm{g} / \mathrm{mL})$ in a $5 \% \mathrm{CO}_{2}$ incubator at $37^{\circ} \mathrm{C}$. The follicle-associated epithelium (FAE) model, mimicking $\mathrm{M}$ cells, was prepared according to des Rieux et al. [28]. Briefly, Caco- 2 cells $\left(1 \times 10^{6}\right.$ cells/well $)$ were cultured on upper inserts for 14 days. Raji B cells $\left(1 \times 10^{6}\right.$ cells/well $)$ in Dulbecco's modified eagle's medium (DMEM; Welgene) were then added to basolateral inserts, and co-cultured for 5 days (trans epithelial electrical resistance (TEER) of 150-200 $\Omega \mathrm{cm}^{2}$ ). Finally, the apical medium of co-culture monolayers was replaced by medium containing particles $(500 \mu \mathrm{g} / \mathrm{mL})$ and further incubated for $6 \mathrm{~h}$. The basolateral solutions were collected and used for quantitative analysis of transported $\mathrm{TiO}_{2}$ particles. 
A Caco-2 monoculture model was used to evaluate particle transport by intestinal epithelial tight junction barrier. Caco-2 cells $\left(4.5 \times 10^{5}\right.$ cells/well) were cultured on upper inserts for 21 days (TEER $\geq 300 \Omega \mathrm{cm}^{2}$ ), and apical medium of the monolayers was replaced by medium containing particles $(500 \mu \mathrm{g} / \mathrm{mL})$, and incubation was continued for $6 \mathrm{~h}$. The basolateral solutions were collected to determine transported $\mathrm{TiO}_{2}$ concentration.

\subsection{Statistical Analysis}

Experimental data were presented as means \pm standard deviations. One-way analysis of variance (ANOVA) followed by Tukey's test in SAS Ver.9.4 (SAS Institute Inc., Cary, NC, USA) was carried out to determine statistical significance at $p$-values $<0.05$.

\section{Results}

\subsection{Characterization of Food Additive $\mathrm{TiO}_{2}$}

Crystal structure of commercially available food additive $\mathrm{E} 171 \mathrm{TiO}_{2}$ particles was determined by XRD patterns. $\mathrm{All}_{\mathrm{TiO}}$ particles were found to be anatase form without significant organic moiety (Figure S1). The SEM images showed irregular and randomly shaped particles, with average primary particle sizes of $118.29 \pm 25.80-169.30 \pm 29.82 \mathrm{~nm}$ and particle size distributions of $1-22 \%$ of smaller than $100 \mathrm{~nm}$ (Figure 1 and Table 1). Statistical differences in average primary particle size among five food additive $\mathrm{TiO}_{2}$ particles were not found $(p>0.05)$. Meanwhile, general-grade nano $\mathrm{TiO}_{2}$ had average particle sizes of $51.45 \pm 11.63$ consisting of $100 \%$ smaller than $100 \mathrm{~nm}$. Whereas, general-grade bulk $\mathrm{TiO}_{2}$ had statistically similar particle sizes compared to food additive $\mathrm{TiO}_{2}$ particles.

T1
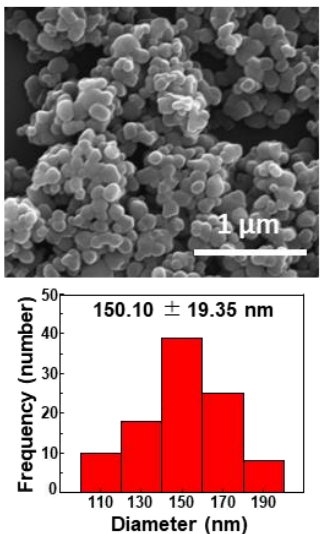

T4
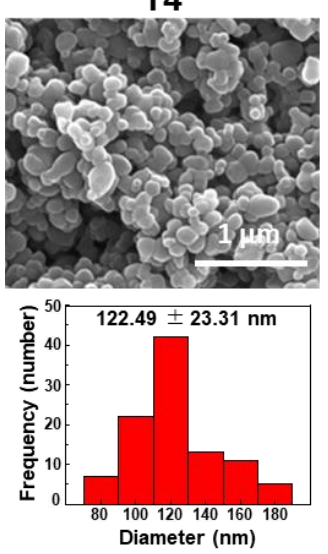

T2
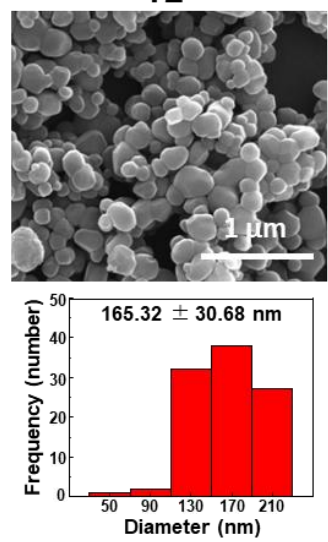

T3
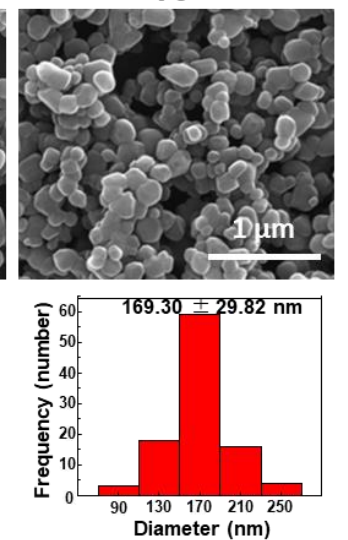

T5
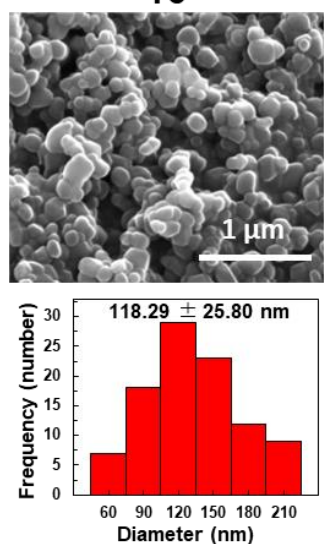

Bulk
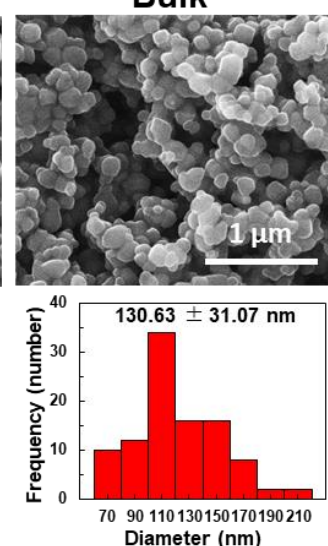
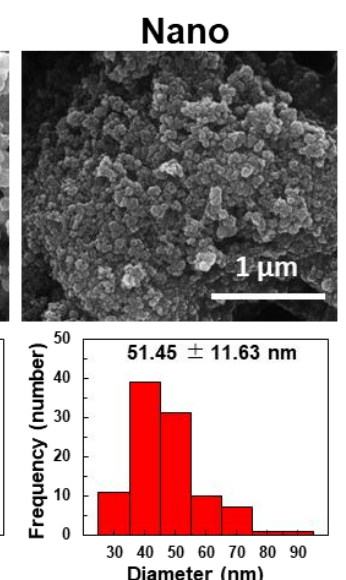

Figure 1. Scanning electron microscopy (SEM) images and size distributions of commercially available food additive $\mathrm{TiO}_{2}$ (T1-T5) and general-grade bulk and nano $\mathrm{TiO}_{2}$ (bulk and nano). Size distributions were obtained by randomly selecting 100 particles from the SEM images. 
Table 1. Primary particle sizes and size distributions of food additive $\mathrm{TiO}_{2}$ (T1-T5) and general-grade bulk and nano $\mathrm{TiO}_{2}$ (bulk and nano) measured by SEM.

\begin{tabular}{ccccc}
\hline \multirow{2}{*}{ Sample } & Average Size $(\mathbf{n m})$ & \multicolumn{3}{c}{ Distribution (No. \%) } \\
\cline { 3 - 5 } & & $\mathbf{<} \mathbf{1 0 0} \mathbf{n m}$ & $\mathbf{1 0 0 - 2 0 0} \mathbf{n m}$ & $>\mathbf{2 0 0} \mathbf{~ n m}$ \\
\hline T1 & $150.10 \pm 19.35^{\mathrm{b}}$ & 1 & 96 & 3 \\
T2 & $165.32 \pm 30.68^{\mathrm{b}}$ & 3 & 80 & 17 \\
T3 & $169.30 \pm 29.82^{\mathrm{b}}$ & 1 & 76 & 23 \\
T4 & $122.49 \pm 23.31^{\mathrm{b}}$ & 12 & 88 & ND \\
T5 & $118.29 \pm 25.80^{\mathrm{ab}}$ & 22 & 74 & 4 \\
Bulk & $130.63 \pm 31.07^{\mathrm{b}}$ & 14 & 84 & 2 \\
Nano & $51.45 \pm 11.63^{\mathrm{a}}$ & 100 & ND & ND
\end{tabular}

Different lowercase letters $(a, b)$ indicate significant differences among different $\mathrm{TiO}_{2}$ particles $(p<0.05)$. No., number; ND, not detectable.

Dynamic light scattering (DLS) results demonstrated that the $\mathrm{Z}$-average sizes of $\mathrm{TiO}_{2}$ particles in DW were $285.30 \pm 6.68-345.27 \pm 4.45 \mathrm{~nm}$, and $100 \%$ of the hydrodynamic diameters of all particles were larger than $100 \mathrm{~nm}$ (Table 2). Thus, the hydrodynamic radii of $\mathrm{TiO}_{2}$ particles in aqueous solution were much larger than the primary particle sizes measured by SEM (Figure 1). Zeta potentials of all food additive $\mathrm{TiO}_{2}$ particles were negative, showing $-36.70 \pm 2.01$ to $-42.17 \pm 3.61 \mathrm{mV}$ (Table 2). On the other hand, size distribution of general-grade bulk $\mathrm{TiO}_{2}$, measured by DLS, was $45.47 \%$ of 100-200 $\mathrm{nm}$ and $54.53 \%$ of larger than $200 \mathrm{~nm}$, without particle size smaller than $100 \mathrm{~nm}$, whereas, general-grade nano $\mathrm{TiO}_{2}$ was composed of $38.27 \%$ of smaller than $100 \mathrm{~nm}, 61.60 \%$ of $100-200 \mathrm{~nm}$, and minimum level $(0.13 \%)$ of larger than $200 \mathrm{~nm}$. Considering average particle sizes and size distributions obtained by SEM and DLS analysis, T3 and T4 were used as representative largest and smallest particles, respectively, for further study.

Table 2. Size distributions, hydrodynamic radii, and zeta potentials of food additive $\mathrm{TiO}_{2}(\mathrm{~T} 1-\mathrm{T} 5)$ and general-grade bulk and nano $\mathrm{TiO}_{2}$ (bulk and nano).

\begin{tabular}{cccccc}
\hline \multirow{2}{*}{ Sample } & \multicolumn{3}{c}{ Distribution (No. \%) } & \multirow{2}{*}{ Z-Average Size (nm) } & Zeta Potential (mV) \\
\cline { 2 - 4 } & $\mathbf{< 1 0 0 ~ n m}$ & $\mathbf{1 0 0 - 2 0 0 ~} \mathbf{~ m}$ & $\mathbf{> 2 0 0} \mathbf{~ n m}$ & & \\
\hline T1 & ND & $44.73 \pm 7.05$ & $55.27 \pm 7.05$ & $285.30 \pm 6.68^{\mathrm{b}}$ & $-40.50 \pm 3.06^{\mathrm{a}}$ \\
T2 & ND & $38.97 \pm 1.32$ & $61.03 \pm 1.32$ & $299.93 \pm 1.79^{\mathrm{b}}$ & $-40.73 \pm 2.46^{\mathrm{a}}$ \\
T3 & ND & $10.43 \pm 1.40$ & $89.57 \pm 1.40$ & $345.27 \pm 4.45^{\mathrm{c}}$ & $-42.17 \pm 1.63^{\mathrm{a}}$ \\
T4 & ND & $49.97 \pm 4.87$ & $50.03 \pm 4.87$ & $291.60 \pm 3.76^{\mathrm{b}}$ & $-36.70 \pm 2.01^{\mathrm{a}}$ \\
T5 & ND & $12.53 \pm 2.61$ & $87.47 \pm 2.61$ & $340.00 \pm 3.94^{\mathrm{c}}$ & $-42.17 \pm 3.61^{\mathrm{a}}$ \\
Bulk & ND & $45.47 \pm 1.70$ & $54.53 \pm 1.70$ & $293.10 \pm 0.52^{\mathrm{b}}$ & $-37.90 \pm 3.38^{\mathrm{a}}$ \\
Nano & $38.27 \pm 18.28$ & $61.60 \pm 18.10$ & $0.13 \pm 0.23$ & $153.43 \pm 11.72^{\mathrm{a}}$ & $13.77 \pm 0.74^{\mathrm{b}}$ \\
\hline
\end{tabular}

Different lowercase letters $(a, b, c)$ indicate significant differences among different particles $(p<0.05)$. No., number; ND, not detectable.

\subsection{Characterization and Quantitative Analysis of $\mathrm{TiO}_{2}$ in Simulated Foods}

In order to check the reliability of analytical procedure for detection of $\mathrm{TiO}_{2}$ in commercial food products, representative $\mathrm{TiO}_{2}$-containing simulated foods, such as sugar powder and chewing gum, were prepared based on the major utilization of $\mathrm{TiO}_{2}$ in foods. The simulated foods were prepared by mixing $\mathrm{TiO}_{2}$ with grinded sugar powder or by attaching $\mathrm{TiO}_{2}$ on the surface of gum base followed by sugar coating. After acid (nitric acid and hydrogen peroxide) treatment for digestion of organic matrices, SEM images showed that $\mathrm{TiO}_{2}$ particles (T3 and T4) recovered from simulated foods had almost the same morphology and particle size distribution compared to pristine ones (Figure 2). The DLS results demonstrated no statistical differences in hydrodynamic radii among pristine $\mathrm{TiO}_{2}$ and $\mathrm{TiO}_{2}$ recovered from simulated foods (Table 3). On the other hand, zeta potential values of $\mathrm{TiO}_{2}$ recovered from simulated foods changed to be fewer negative charges compared to pristine $\mathrm{TiO}_{2}$ (Table 3). 


\section{Simulated foods}
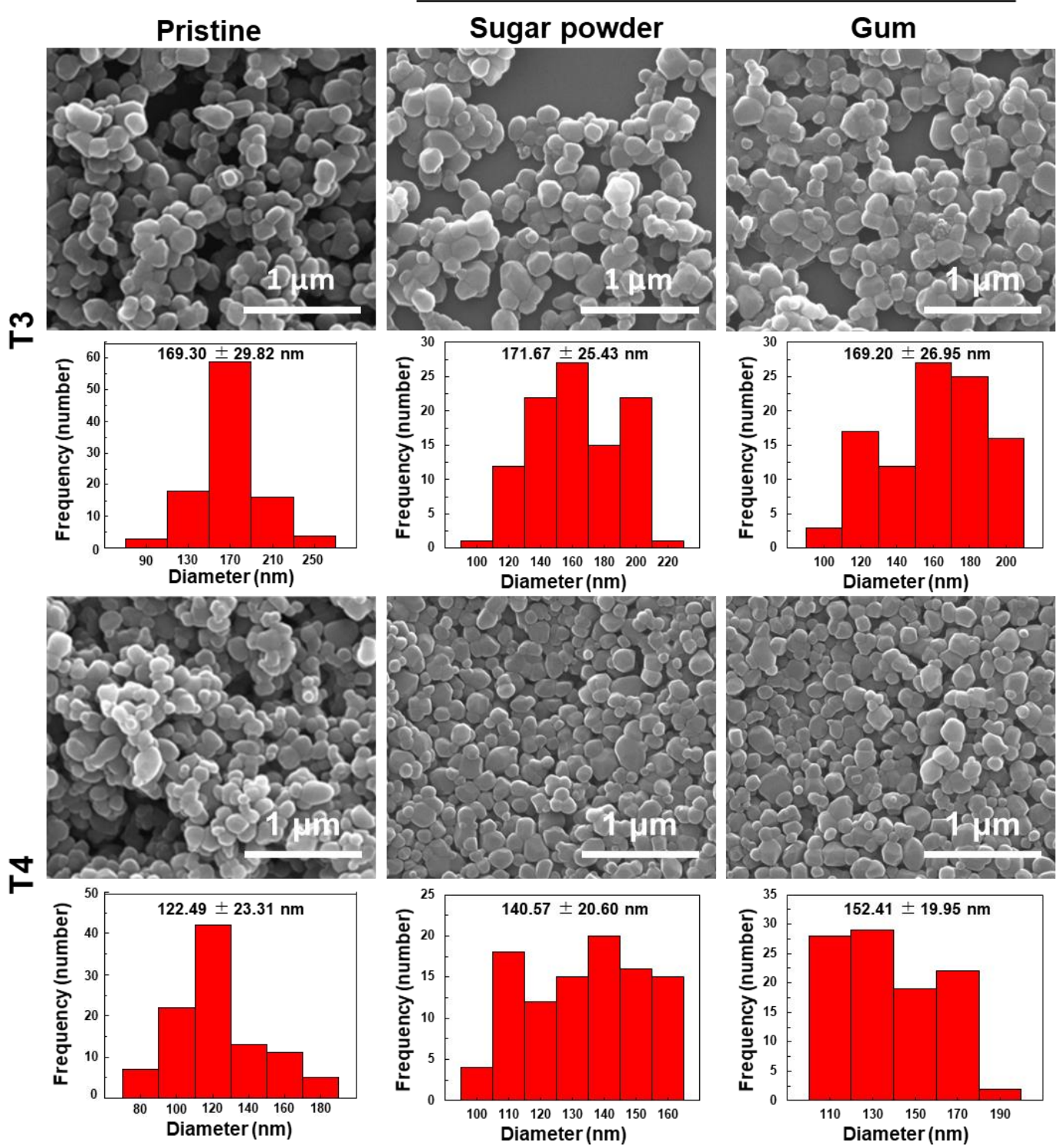

Figure 2. Scanning electron microscopy (SEM) images and size distributions of food additive $\mathrm{TiO}_{2}(\mathrm{~T} 3$ and T4) recovered from simulated foods. Size distributions were obtained by randomly selecting 100 particles from SEM images.

Table 3. Size distributions, hydrodynamic radii, and zeta potentials of food additive $\mathrm{TiO}_{2}$ ( $\mathrm{T} 3$ and $\mathrm{T} 4$ ) recovered from simulated foods.

\begin{tabular}{|c|c|c|c|c|c|c|}
\hline & \multirow{2}{*}{ Sample } & \multicolumn{3}{|c|}{ Distribution (No. \%) } & \multirow{2}{*}{ Z-Average Size (nm) } & \multirow{2}{*}{ Zeta Potential (mV) } \\
\hline & & $<100 \mathrm{~nm}$ & $100-200 \mathrm{~nm}$ & $>200 \mathrm{~nm}$ & & \\
\hline \multirow{3}{*}{ T3 } & Pristine & ND & $10.43 \pm 1.40$ & $89.57 \pm 1.40$ & $345.27 \pm 4.45^{\mathrm{a}}$ & $-42.17 \pm 1.63^{a}$ \\
\hline & Sugar powder & ND & $37.03 \pm 13.22$ & $62.97 \pm 13.22$ & $327.30 \pm 14.90^{\mathrm{a}}$ & $-21.77 \pm 6.61^{b}$ \\
\hline & Gum & ND & $24.97 \pm 3.39$ & $75.03 \pm 3.39$ & $332.30 \pm 5.20^{a}$ & $-17.37 \pm 1.99^{b}$ \\
\hline \multirow{3}{*}{ T4 } & Pristine & ND & $49.97 \pm 4.87$ & $50.03 \pm 4.87$ & $291.60 \pm 3.76^{a}$ & $-36.70 \pm 2.04^{a}$ \\
\hline & Sugar powder & $0.23 \pm 0.40$ & $36.83 \pm 26.04$ & $62.93 \pm 26.28$ & $293.27 \pm 16.69^{a}$ & $-37.67 \pm 3.48^{a}$ \\
\hline & Gum & ND & $40.07 \pm 17.56$ & $59.93 \pm 17.56$ & $315.40 \pm 2.46^{a}$ & $-16.57 \pm 1.12^{b}$ \\
\hline
\end{tabular}

Different lowercase letters $(a, b)$ indicate significant differences between pristine $\mathrm{TiO}_{2}$ and $\mathrm{TiO}_{2}$ recovered from simulated foods $(p<0.05)$. No., number; ND, not detectable. 
Standard calibration curves of $\mathrm{TiO}_{2}$ ( $\mathrm{T} 3$ and $\left.\mathrm{T} 4\right)$ in the simulated foods were obtained after acid digestion of food matrices, dissolution of $\mathrm{TiO}_{2}$ in sulfuric acid, followed by Ti quantification by ICP-AES. In this step, sulfuric acid was used to dissolve Ti from $\mathrm{TiO}_{2}[29,30]$. Figure 3 showed that there were no significant differences in Ti quantification between pristine $\mathrm{TiO}_{2}$ and sugar powder- or gum base-spiked $\mathrm{TiO}_{2}$, with linear correlation coefficients. The recoveries (\%) of $\mathrm{TiO}_{2}$ were more than $90 \%$ in all cases, regardless of particle size distribution (T3 and T4) and food matrix type (Table 4). The limits of detection (LOD) and limits of quantification (LOQ) for pristine $\mathrm{TiO}_{2}$ as well as $\mathrm{TiO}_{2}$ in simulated foods were sufficiently low to detect the particles in food matrices.
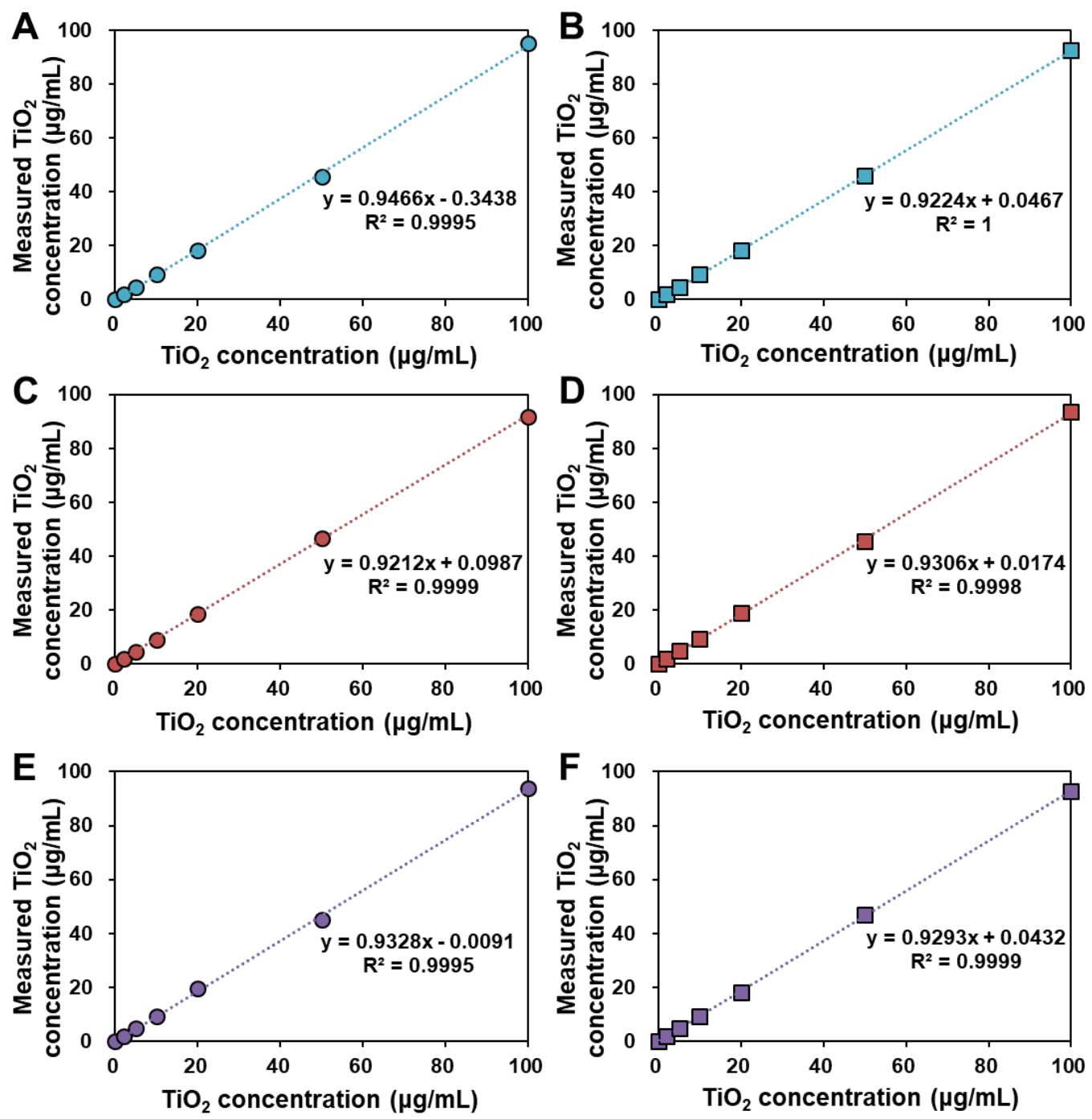

Figure 3. Calibration curves for determination of (A) pristine $\mathrm{T} 3$ and (B) pristine $\mathrm{T} 4,(\mathbf{C}) \mathrm{T} 3$ and (D) T4 in simulated sugar powder, and (E) T3 and (F) T4 in simulated gum by inductively coupled plasma-atomic emission spectroscopy (ICP-AES) analysis. 
Table 4. Analytical recovery, limit of detection (LOD), and limit of quantification (LOQ) of quantitative analytical procedure for $\mathrm{TiO}_{2}$.

\begin{tabular}{|c|c|c|c|c|c|c|c|c|}
\hline \multirow{2}{*}{ Sample } & & \multirow{2}{*}{ Parameters } & \multicolumn{6}{|c|}{$\mathrm{TiO}_{2}$ Concentration $(\mu \mathrm{g} / \mathrm{mL})$} \\
\hline & & & 2 & 5 & 10 & 20 & 50 & 100 \\
\hline \multirow{6}{*}{ Pristine } & & Recovery (\%) & $93.42 \pm 2.78$ & $93.86 \pm 7.43$ & $92.14 \pm 3.53$ & $91.34 \pm 1.52$ & $90.88 \pm 5.72$ & $95.12 \pm 1.52$ \\
\hline & T3 & $\mathrm{LOD}(\mu \mathrm{g} / \mathrm{mL})$ & \multirow{2}{*}{\multicolumn{6}{|c|}{$\begin{array}{l}0.05 \\
0.15\end{array}$}} \\
\hline & & $\mathrm{LOQ}(\mu \mathrm{g} / \mathrm{mL})$ & & & & & & \\
\hline & \multirow{3}{*}{$\mathrm{T} 4$} & Recovery (\%) & $102.08 \pm 1.67$ & $93.31 \pm 4.60$ & $95.09 \pm 4.66$ & $91.18 \pm 0.57$ & $91.88 \pm 6.39$ & $92.43 \pm 2.54$ \\
\hline & & $\mathrm{LOD}(\mu \mathrm{g} / \mathrm{mL})$ & \multirow{2}{*}{\multicolumn{6}{|c|}{$\begin{array}{l}0.23 \\
0.70\end{array}$}} \\
\hline & & $\mathrm{LOQ}(\mu \mathrm{g} / \mathrm{mL})$ & & & & & & \\
\hline \multirow{6}{*}{$\begin{array}{c}\text { Simulated } \\
\text { sugar powder }\end{array}$} & \multirow{3}{*}{$\mathrm{T} 3$} & Recovery (\%) & $96.81 \pm 3.48$ & $93.19 \pm 10.98$ & $90.85 \pm 8.49$ & $92.72 \pm 3.49$ & $93.65 \pm 4.24$ & $91.91 \pm 2.80$ \\
\hline & & $\mathrm{LOD}(\mu \mathrm{g} / \mathrm{mL})$ & \multirow{2}{*}{\multicolumn{6}{|c|}{$\begin{array}{l}0.34 \\
1.04\end{array}$}} \\
\hline & & LOQ $(\mu \mathrm{g} / \mathrm{mL})$ & & & & & & \\
\hline & \multirow{3}{*}{$\mathrm{T} 4$} & Recovery (\%) & $99.15 \pm 2.64$ & $97.96 \pm 3.59$ & $92.12 \pm 3.96$ & $95.01 \pm 2.57$ & $91.10 \pm 7.21$ & $93.50 \pm 3.67$ \\
\hline & & $\mathrm{LOD}(\mu \mathrm{g} / \mathrm{mL})$ & \multirow{2}{*}{\multicolumn{6}{|c|}{$\begin{array}{l}0.18 \\
0.55\end{array}$}} \\
\hline & & $\mathrm{LOQ}(\mu \mathrm{g} / \mathrm{mL})$ & & & & & & \\
\hline \multirow{6}{*}{$\begin{array}{l}\text { Simulated } \\
\text { gum }\end{array}$} & & Recovery (\%) & $93.96 \pm 4.89$ & $93.96 \pm 3.50$ & $93.41 \pm 3.01$ & $97.72 \pm 2.34$ & $90.03 \pm 5.48$ & $93.90 \pm 0.64$ \\
\hline & T3 & $\mathrm{LOD}(\mu \mathrm{g} / \mathrm{mL})$ & \multirow{2}{*}{\multicolumn{6}{|c|}{$\begin{array}{l}0.03 \\
0.09\end{array}$}} \\
\hline & & $\mathrm{LOQ}(\mu \mathrm{g} / \mathrm{mL})$ & & & & & & \\
\hline & & Recovery (\%) & $97.58 \pm 3.64$ & $96.30 \pm 3.98$ & $93.74 \pm 2.35$ & $90.54 \pm 1.16$ & $94.04 \pm 2.82$ & $92.81 \pm 1.88$ \\
\hline & $\mathrm{T} 4$ & $\mathrm{LOD}(\mu \mathrm{g} / \mathrm{mL})$ & \multicolumn{6}{|c|}{0.21} \\
\hline & & LOQ $(\mu \mathrm{g} / \mathrm{mL})$ & \multicolumn{6}{|c|}{0.62} \\
\hline
\end{tabular}

\subsection{Characterization and Quantitative Analysis of $\mathrm{TiO}_{2}$ in Commercial Foods}

Food additive $\mathrm{TiO}_{2}$ particles were characterized and identified from various commercial products, such as candy, chocolate, chewing gum, jelly, sauces, and snacks, based on the method established with the simulated foods. The SEM images revealed that $\mathrm{TiO}_{2}$ particles recovered from commercial foods by nitric acid/hydrogen peroxide treatment had similar shape and particle size distribution (Figure 4) without significant decomposition compared to pristine $\mathrm{TiO}_{2}$ (Figure 1). The DLS results showed that the hydrodynamic radii of $\mathrm{TiO}_{2}$ particles recovered from commercial products were almost similar, ranging from $242.53 \pm 45.60-461.47 \pm 6.76 \mathrm{~nm}$ (Table 5), compared to those of pristine (Table 2). Zeta potential changes to less negative charges were also observed (Table 5), as did in simulated foods (Table 3). Element analysis using EDS confirmed the presence of Ti in the particles recovered from commercial foods (Figure S2).

Table 5. Size distributions, hydrodynamic radii, zeta potentials, and quantitative results of $\mathrm{TiO}_{2}$ recovered from commercial foods.

\begin{tabular}{ccccccc}
\hline \multirow{2}{*}{$\begin{array}{c}\text { Commercial } \\
\text { Products }\end{array}$} & \multicolumn{3}{c}{ Distribution (No. \%) } & \multirow{2}{*}{ Z-Average Size (nm) } & Zeta Potential (mV) & TiO $\mathbf{2}$ Contents $(\mathbf{m g} / \mathbf{g})$ \\
\cline { 2 - 4 } & $\mathbf{<} \mathbf{1 0 0} \mathbf{~ n m}$ & $\mathbf{1 0 0 - 2 0 0 ~} \mathbf{~ n m}$ & $\mathbf{> 2 0 0} \mathbf{~ n m}$ & & & \\
\hline Candy 1 & ND & ND & $100.00 \pm 0.00$ & $417.80 \pm 25.06$ & $-35.37 \pm 2.03$ & $1.09 \pm 0.03$ \\
Candy 2 & $3.83 \pm 6.64$ & $39.67 \pm 25.52$ & $56.50 \pm 31.92$ & $306.83 \pm 59.91$ & $-13.13 \pm 0.45$ & $9.87 \pm 0.21$ \\
Chocolate 1 & $2.10 \pm 3.64$ & $25.93 \pm 37.80$ & $71.97 \pm 35.75$ & $242.53 \pm 45.60$ & $-12.03 \pm 2.83$ & $8.63 \pm 0.04$ \\
Chocolate 2 & ND & $27.03 \pm 13.11$ & $72.97 \pm 13.11$ & $264.97 \pm 14.55$ & $-31.87 \pm 1.50$ & $6.32 \pm 0.63$ \\
Gum & $11.73 \pm 16.82$ & $47.50 \pm 14.81$ & $40.77 \pm 22.17$ & $344.90 \pm 4.78$ & $-14.50 \pm 0.62$ & $3.53 \pm 0.12$ \\
Jelly 1 & $0.90 \pm 1.56$ & $35.47 \pm 27.51$ & $63.63 \pm 28.93$ & $363.17 \pm 11.29$ & $-37.23 \pm 0.72$ & $1.30 \pm 0.13$ \\
Jelly 2 & ND & $17.43 \pm 23.88$ & $82.57 \pm 23.88$ & $298.83 \pm 82.98$ & $-32.57 \pm 0.76$ & $2.33 \pm 0.37$ \\
Sauce 1 & ND & $63.40 \pm 34.26$ & $36.60 \pm 34.26$ & $316.07 \pm 35.30$ & $-25.20 \pm 1.15$ & $3.70 \pm 0.41$ \\
Sauce 2 & ND & $11.33 \pm 17.76$ & $88.67 \pm 17.76$ & $461.47 \pm 6.76$ & $-36.80 \pm 0.78$ & $0.94 \pm 0.04$ \\
Snack & ND & $2.87 \pm 4.13$ & $97.13 \pm 4.13$ & $356.63 \pm 8.45$ & $-25.93 \pm 0.47$ & $1.27 \pm 0.08$ \\
\hline
\end{tabular}

No., number; ND, not detectable. 

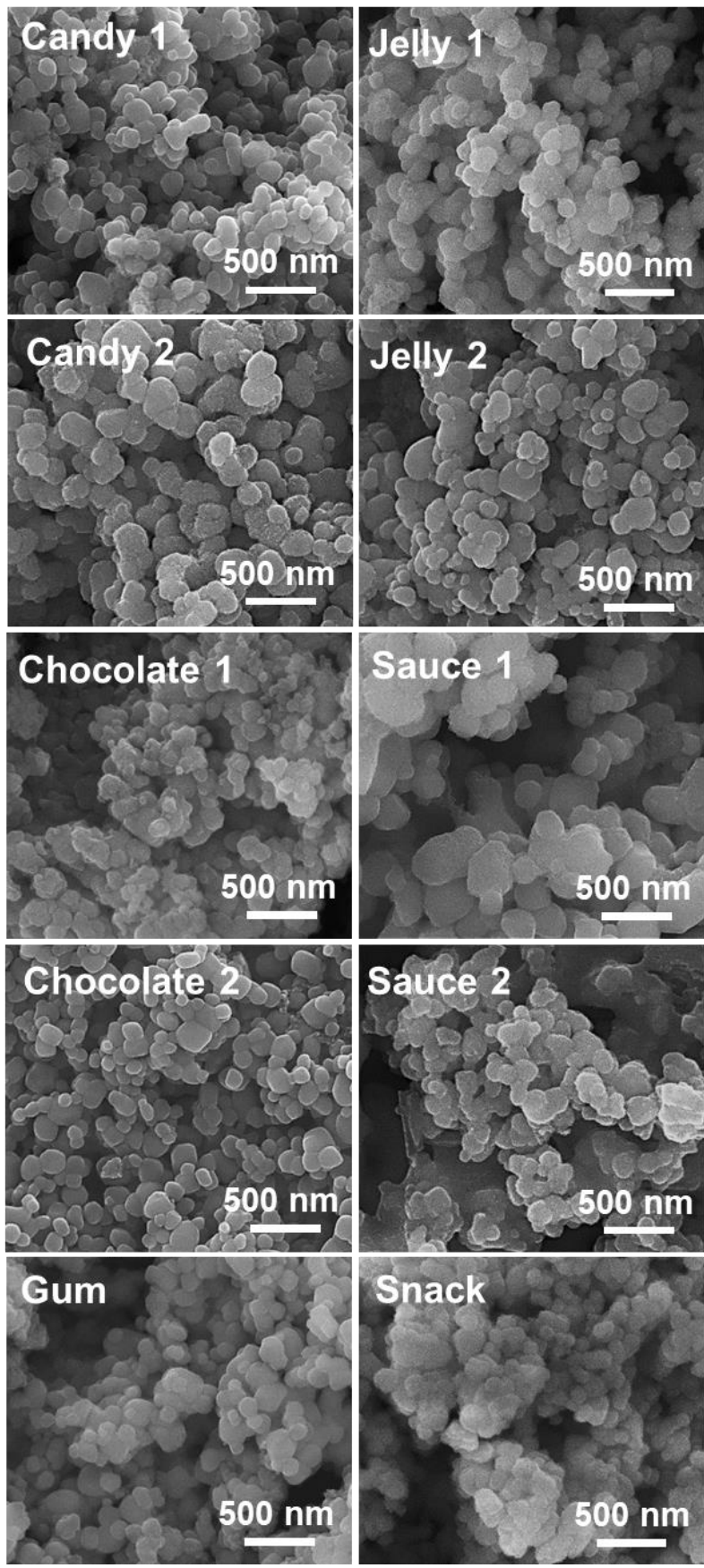

Figure 4. Scanning electron microscopy (SEM) images of $\mathrm{TiO}_{2}$ particles recovered from commercial foods.

On the other hand, quantitative analysis revealed that $\mathrm{TiO}_{2}$ contents ranged from $1.09-9.87 \mathrm{mg} / \mathrm{g}$, representing $0.11-0.99 \%(\mathrm{wt} / \mathrm{wt}$ ) (Table 5).

\subsection{Size Fractionation of $\mathrm{TiO}_{2}$}

Size fractionation was performed with the smallest particle T4 using sucrose gradient solution in order to obtain smaller particle size. Figure 5 demonstrated that the top layer of T4 after sucrose gradient fractionation had smaller size with narrower size distribution $(96.94 \pm 17.86 \mathrm{~nm}$, Table S1) than pristine $\mathrm{T} 4(122.49 \pm 23.31 \mathrm{~nm})$, and the particle size increased in the bottom layer. The homogeneity of size distribution was checked by kurtosis values from normal distribution fitting (Table S1). Kurtosis value for the top layer was 1.11, more positive than the value (1.07) for pristine T4, indicating narrower 
size distribution of the former than the latter. Thus, the top layer of $\mathrm{T} 4$ after size fractionation was used for further cytotoxicity study.

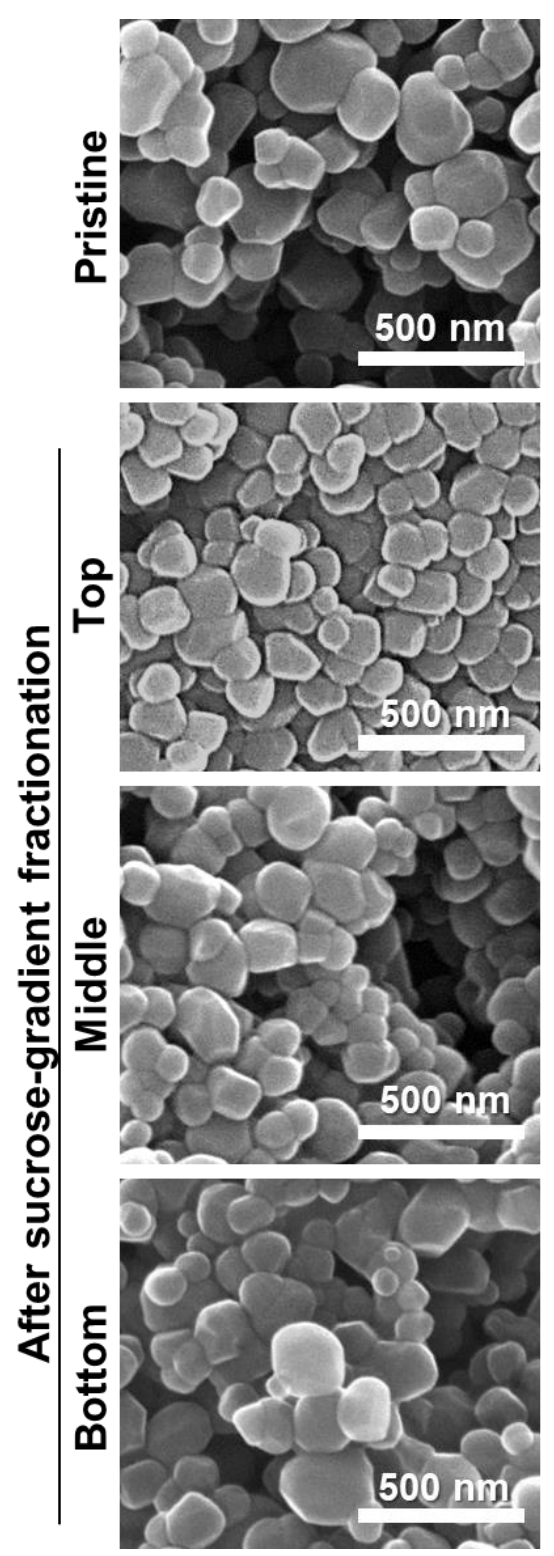

Figure 5. Scanning electron microscopy (SEM) images of $\mathrm{TiO}_{2}$ (T4) in the top, middle, and bottom layers fractionated by a sucrose gradient method.

\subsection{Inhibition of Cell Proliferation, Membrane Damage, and Colony Formation}

The effects of food additive $\mathrm{TiO}_{2}$ with different size distributions ( $\mathrm{T} 3$ and T4) on short-term cell proliferation and membrane damage were evaluated in human intestinal Caco- 2 cells after $24 \mathrm{~h}$ exposure with WST-1 and lactate dehydrogenase (LDH) leakage assays, respectively. General-grade bulk and nano $\mathrm{TiO}_{2}$ were also used for comparative study. The results demonstrated that all food-grade and general-grade $\mathrm{TiO}_{2}$ particles did not affect cell proliferation or membrane integrity after $24 \mathrm{~h}$ (Figure 6A,B). 

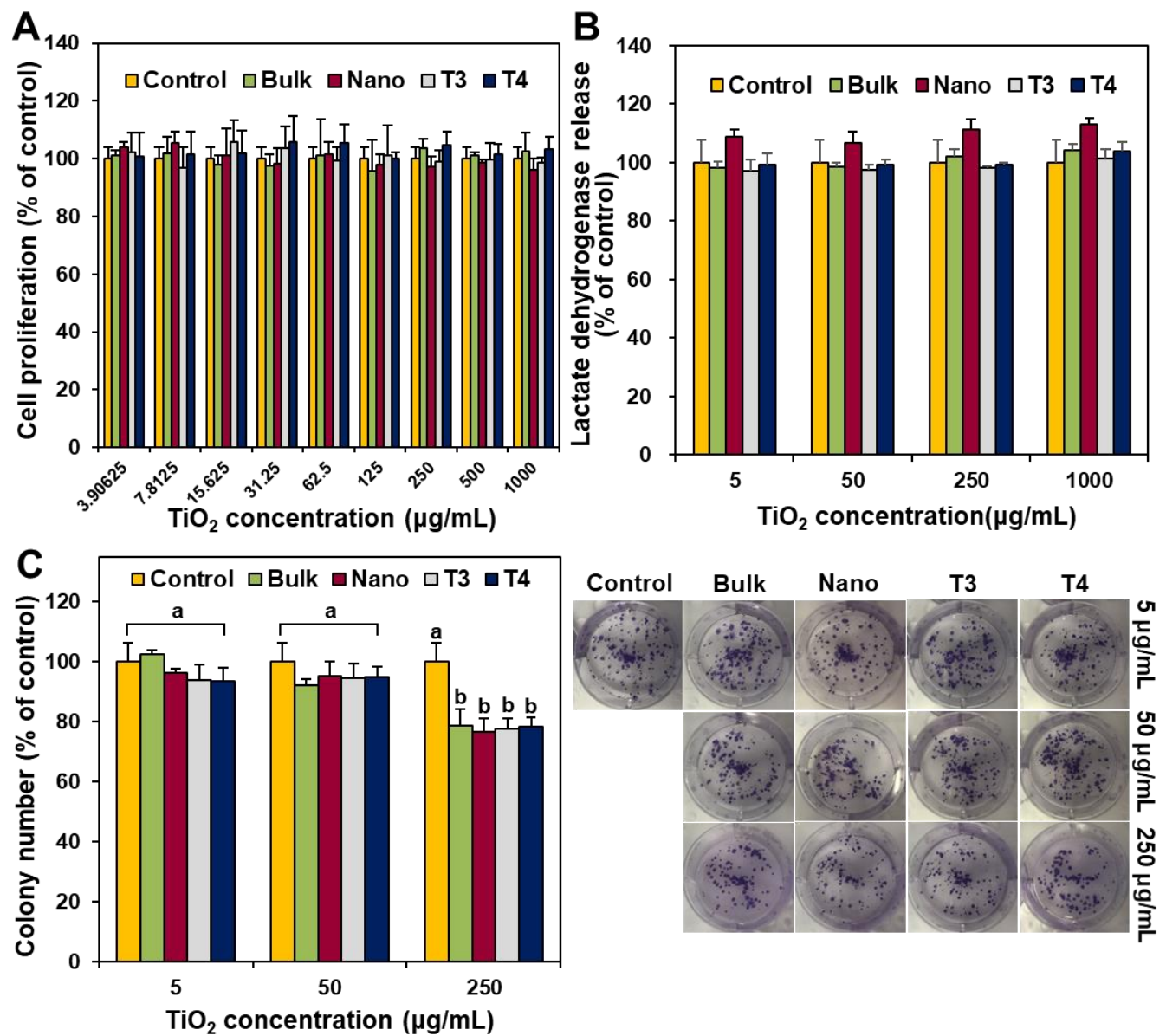

Figure 6. Short-term effect of $\mathrm{TiO}_{2}$ particles on (A) cell proliferation and (B) membrane damage of human intestinal Caco-2 cells after $24 \mathrm{~h}$. (C) Long-term effect of $\mathrm{TiO}_{2}$ particles on colony-forming ability of Caco-2 cells after 14 days. Different lowercase letters $(a, b)$ indicate significant differences among control (cells without particle) and different $\mathrm{TiO}_{2}$ particles (food-grade $\mathrm{T} 3$, $\mathrm{T} 4$, general-grade bulk and nano) $(p<0.05)$.

The long-term effect of food additive $\mathrm{TiO}_{2}$ on colony formation was also investigated with clonogenic assay and compared with general-grade bulk and nano $\mathrm{TiO}_{2}$. Figure $6 \mathrm{C}$ showed that all $\mathrm{TiO}_{2}$ particles slightly inhibited colony-forming ability at the highest concentration tested $(250 \mu \mathrm{g} / \mathrm{mL})$ after exposure for 14 days, regardless of particle size distribution or type.

When the effect of the smallest particle fraction of T4 (Figure 5), the top layer after size fractionation using sucrose gradient solution, on short-term cell proliferation, was evaluated with the WST-1 assay, no significant differences in cytotoxicity were found between pristine T4 and size-fractioned top layer of T4 (Figure 7). Cell culture medium or T4 containing 3.2\% sucrose (the remaining sucrose concentration after size fractionation) was also used as controls to confirm no effect of the sucrose on cytotoxicity.

\subsection{ROS and Apoptosis Induction}

The potential of food additive $\mathrm{TiO}_{2}$ ( $\mathrm{T} 3$ and $\mathrm{T} 4$ ) on oxidative stress induction was evaluated by measuring intracellular ROS. Apoptosis induction was also identified with annexin V-FITC, which binds to the phosphatidylserine exposed at cell surface, that represents an early marker of apoptosis. PI, a dye which is not taken up by the viable cells, was used as a dead cell marker. The results showed that all food-grade $\mathrm{TiO}_{2}$ and general-grade bulk and nano $\mathrm{TiO}_{2}$ significantly induced $\mathrm{ROS}$ at above $125 \mu \mathrm{g} / \mathrm{mL}$ after $24 \mathrm{~h}$ exposure (Figure 8A). No significant differences in ROS generation were found among different types of particles $(p>0.05)$. 


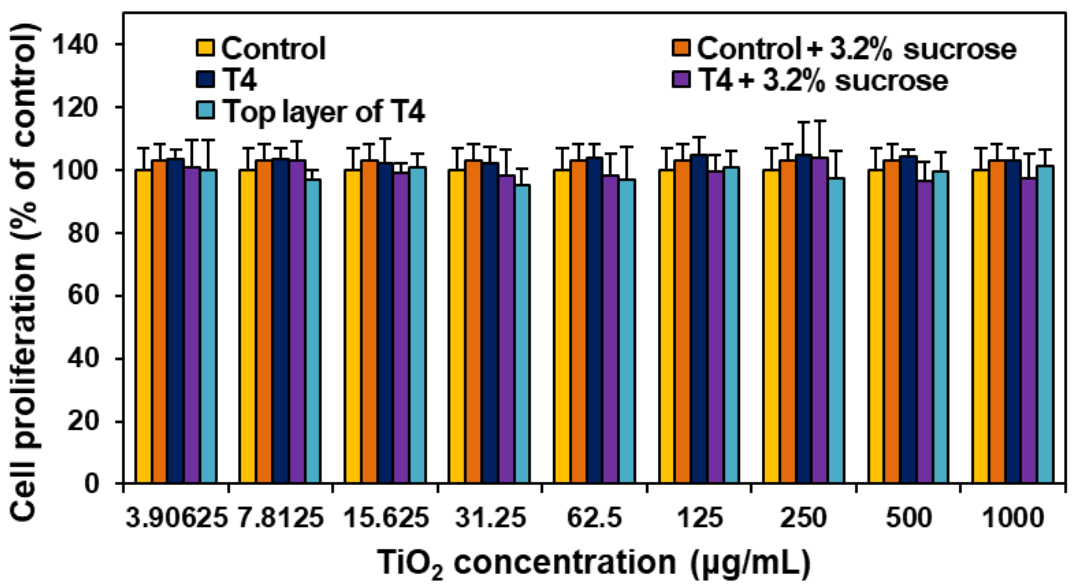

Figure 7. The effect of the smallest particle fraction of $\mathrm{TiO}_{2}(\mathrm{~T} 4)$, the top layer after size fractionation, on cell proliferation of Caco- 2 cells after $24 \mathrm{~h}$. No significant differences among controls (cells without particles), T4, and top layer of T4 were found ( $p>0.05)$.
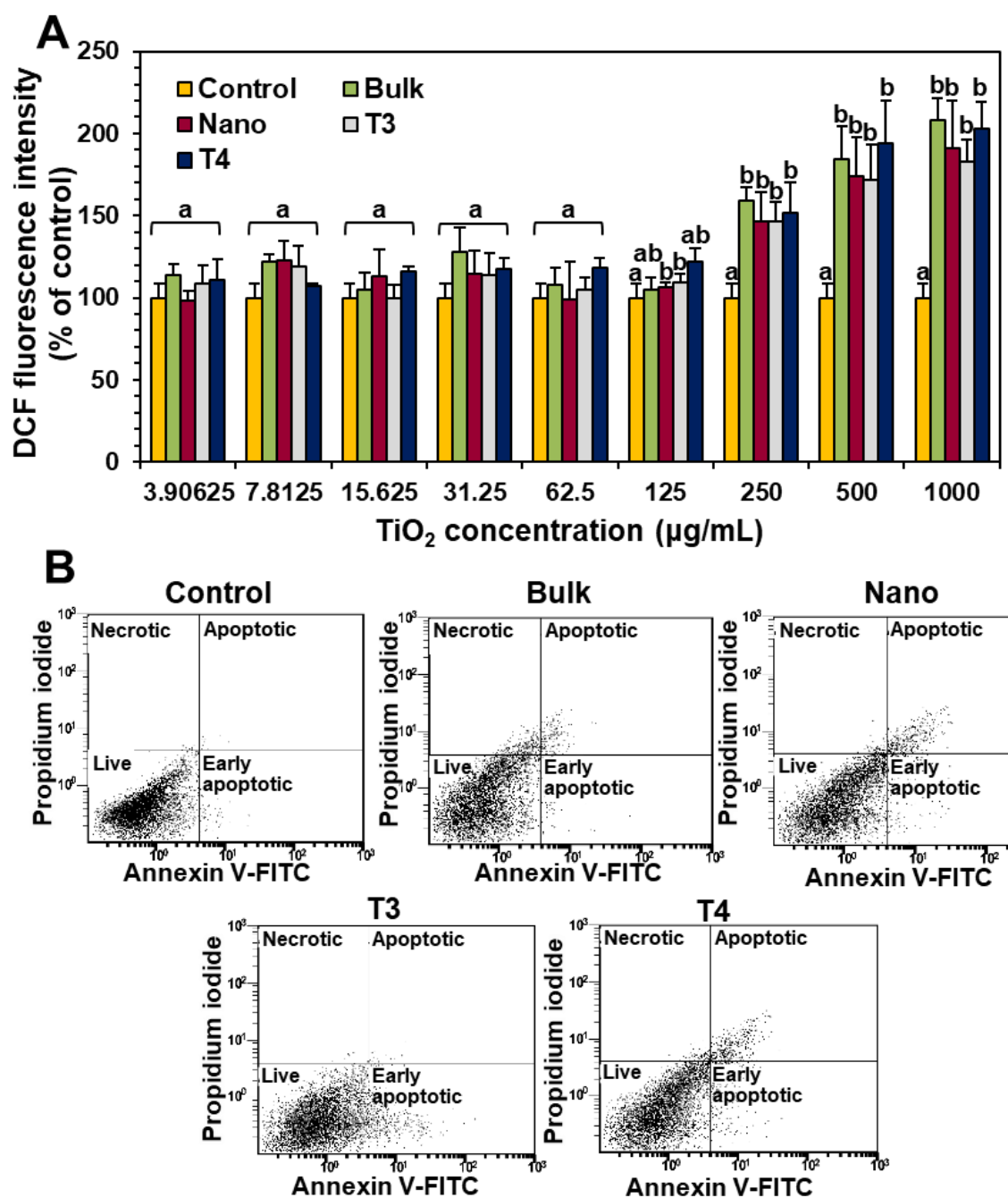

Figure 8. The effect of $\mathrm{TiO}_{2}$ particles on (A) intracellular reactive oxygen species (ROS) generation and (B) apoptosis induction in Caco-2 cells after $24 \mathrm{~h}$. Different lowercase letters (a, b) indicate significant differences among control (cells without particles) and different $\mathrm{TiO}_{2}$ particles (food-grade $\mathrm{T} 3, \mathrm{~T} 4$, general-grade bulk and nano) $(p<0.05)$. 
On the other hand, all $\mathrm{TiO}_{2}$ particles slightly caused apoptosis or necrosis at the highest concentration tested, $1000 \mu \mathrm{g} / \mathrm{mL}$ after exposure for $24 \mathrm{~h}$ (Figure 8B), showing total apoptotic or necrotic cells of $\sim 7.9,11.9,6.8$, and $12.5 \%$ for general-grade bulk, general-grade nano, $\mathrm{T} 3$, and $\mathrm{T}_{4} \mathrm{TiO}_{2}$ particles, respectively.

\subsection{Intestinal Transport Mechanism}

Particles must be transported across the intestinal epithelial barrier to be absorbed. The intestinal transport mechanism of food additive $\mathrm{TiO}_{2}$ ( $\mathrm{T} 3$ and $\mathrm{T} 4$ ) and general-grade bulk and nano $\mathrm{TiO}_{2}$ was investigated by two different culture models: a 2D model, the Caco-2 monolayer, and a 3D one, the FAE. The former and the latter represent microfold (M) cells found in FAE overlying Peyer's patches and intestinal tight junction, respectively. The results demonstrated that all particles were primarily transported by $\mathrm{M}$ cells, but significantly increased transports through Caco-2 monolayer were also found (Figure 9A). The total transport amounts of all $\mathrm{TiO}_{2}$ particles, combined transportation by both $\mathrm{M}$ cells and the Caco- 2 monolayer, was extremely low, showing $0.12-0.14 \%$ (Figure $9 \mathrm{~B}$ ). The effects of particle size or particle type (food- or general-grade) on intestinal transport amount and mechanism were not found $(p>0.05)$.
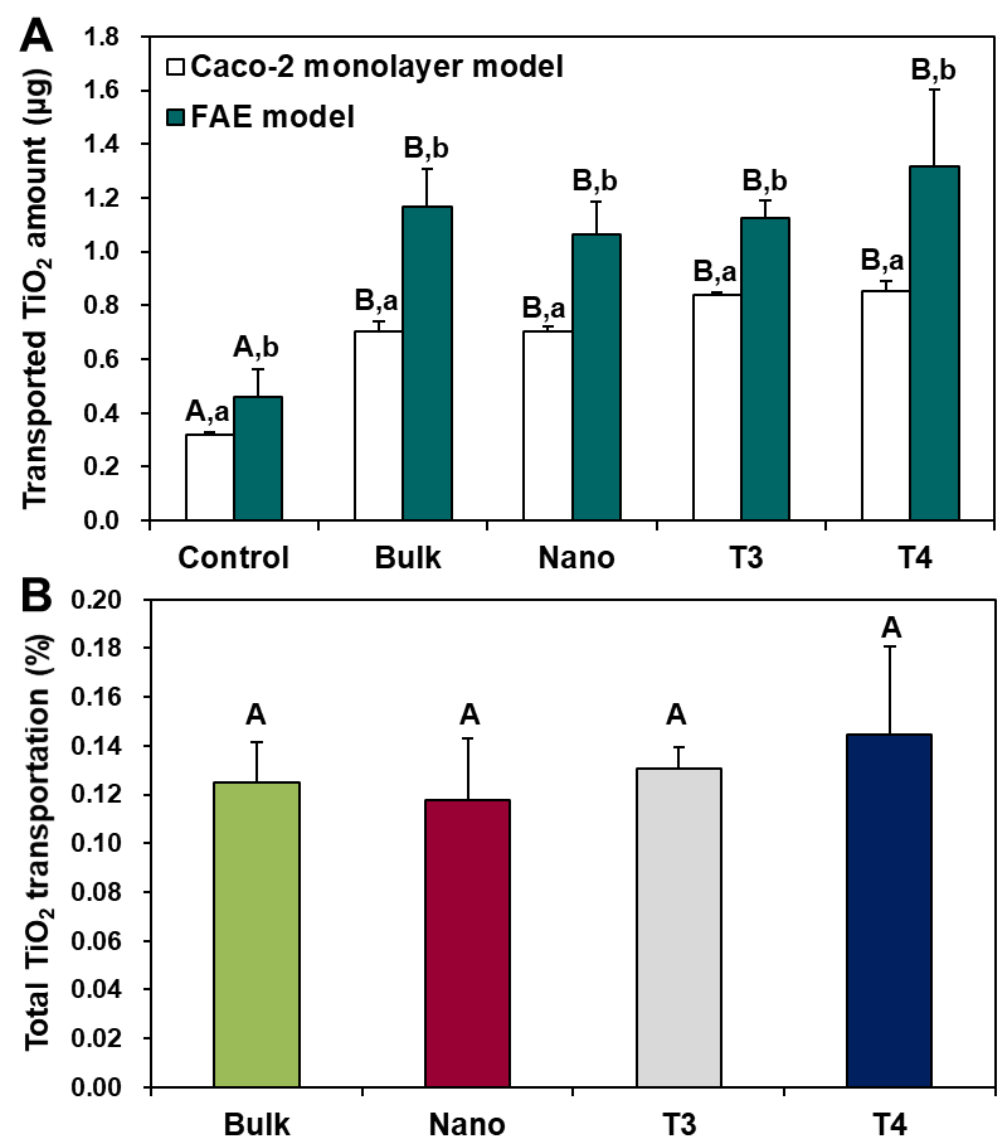

Figure 9. Intestinal transport mechanism of $\mathrm{TiO}_{2}$ particles using $(\mathbf{A})$ in vitro models of $\mathrm{Caco}-2$ monolayer and human follicle-associated epithelium (FAE). (B) Total combined intestinal transport amount of $\mathrm{TiO}_{2}$ particles through both Caco-2 monolayer and FAE models. Different uppercase letters (A, B) indicate significant differences among control (cells without particles) and different $\mathrm{TiO}_{2}$ particles (food-grade $\mathrm{T} 3, \mathrm{~T} 4$, general-grade bulk and nano) $(p<0.05)$. Different lowercase letters $(\mathrm{a}, \mathrm{b})$ indicate significant differences between Caco-2 monolayer and FAE models $(p<0.05)$. 


\section{Discussion}

Titanium dioxide is a widely utilized food additive in the food industry. Hence, the characterization of food additive $\mathrm{TiO}_{2}$ and its determination in commercial foods are of importance, which can answer the fundamental question as to whether food additive $\mathrm{TiO}_{2}$ particles consist of nano-sized materials or not. In the present study, commercially available food additive E171 $\mathrm{TiO}_{2}$ particles were found to be composed of particle sizes of mainly larger than $100 \mathrm{~nm}$, showing 1-22\% smaller than $100 \mathrm{~nm}$ in number-size distribution, based on SEM images (Figure 1 and Table 1). On the other hand, DLS results demonstrated that the hydrodynamic diameters of all food additive $\mathrm{TiO}_{2}$ particles were larger than $100 \mathrm{~nm}$ and that the hydrodynamic radii increased in DW (Table 2) compared to primary particle sizes obtained by SEM analysis (Figure 1). This result suggests that $\mathrm{TiO}_{2}$ particles were present as agglomerated or aggregated forms under aqueous condition. Taken together, $\mathrm{TiO}_{2}$ particles are not considered as nanomaterials according to European Commission Recommendation 2011/696/EU [8].

For accurate and reliable $\mathrm{TiO}_{2}$ determination and quantification in commercial foods, we first established the analytical method using representative $\mathrm{TiO}_{2}$-containing simulated foods, such as sugar powder and gum. Nitric acid and hydrogen peroxide treatment was performed to digest organic food matrices [31,32], which permits physicochemical characterization of intact $\mathrm{TiO}_{2}$ particles after recovery. The results demonstrated almost similar shape and particle size of $\mathrm{TiO}_{2}$ recovered from the simulated foods compared to pristine $\mathrm{TiO}_{2}$ (Figure 2 and Table 3). This indicates that nitric acid/hydrogen peroxide digestion did not significantly alter the morphology and particle size distribution of $\mathrm{TiO}_{2}$. It is known that $\mathrm{TiO}_{2}$ is resistant to acids, except concentrated sulfuric acid [29,30]. Zeta potential changes of $\mathrm{TiO}_{2}$ to fewer negative values after recovery from the simulated foods are strongly likely to be related to acid treatment. It was reported that nitric acid-treated $\mathrm{TiO}_{2}$ particles are positively charged by adsorbing nitric acid on the surface [33,34]. Although nitric acid was evaporated by heating, and then, $\mathrm{TiO}_{2}$ particles were dispersed in DW or ethanol for physicochemical characterization, it is probable that a small portion of nitric acid remained, affecting the zeta potential values. Meanwhile, sulfuric acid treatment was used for Ti dissolution from $\mathrm{TiO}_{2}$, which contributes to Ti quantification by ICP-AES [29,30,35,36]. Figure 3 and Table 4 clearly showed that nitric acid/hydrogen peroxide/sulfuric acid pretreatment can be used as an accurate and precise method for $\mathrm{TiO}_{2}$ quantification.

When the above method was applied to recover $\mathrm{TiO}_{2}$ particles from diverse commercial foods, almost similar morphology and particle size compared to pristine $\mathrm{TiO}_{2}$ were observed (Figure 4), although slightly increased hydrodynamic radii of $\mathrm{TiO}_{2}$ were found in some cases (Table 5). These results suggest that $\mathrm{TiO}_{2}$ particles were present as intact particle forms but could be more agglomerated or aggregated to some extent in food matrices. It is worth noting that the presence of Ti in the particles recovered from commercial products was confirmed by EDS analysis (Figure S2). Based on the results obtained by pristine $\mathrm{TiO}_{2}, \mathrm{TiO}_{2}$-containing simulated foods, and $\mathrm{TiO}_{2}$-containing commercial foods, $\mathrm{TiO}_{2}$ is a material primarily composed of larger than $100 \mathrm{~nm}$ and present as an agglomerated or aggregated particle in aqueous solution or food matrices. On the other hand, quantitative analysis revealed that less than $1 \%(\mathrm{wt} / \mathrm{wt}) \mathrm{TiO}_{2}$ was detected in all commercial food products, which is well correlated to the FDA recommendation [7].

$\mathrm{TiO}_{2}$ particles (T3 and T4) did not cause short-term inhibition of cell proliferation (Figure 6A) or membrane damage (Figure 6B), but slightly induced apoptosis (Figure 8B) after 24 h exposure, as general-grade bulk or nano $\mathrm{TiO}_{2}$ did. When further cytotoxicity was evaluated with smaller and narrower size distribution of T4 after sucrose gradient fractionation (Figure 5 and Table S1), no effect on short-term cell proliferation was also found (Figure 7). However, long-term colony formation was inhibited by high concentration $(250 \mu \mathrm{g} / \mathrm{mL})$ of all $\mathrm{TiO}_{2}$ particles after exposure for 14 days (Figure 6C). Although long-term exposure time and high concentration were used in the point of view of digestion physiology, particle accumulation at target specific organs for prolonged time could not be completely excluded. Nevertheless, it is worth noting that the concentrations exhibiting cytotoxicity are too high to be taken up by food-additive $\mathrm{TiO}_{2}$ in commercial products, considering its extremely low oral absorption. On the other hand, $\mathrm{TiO}_{2}$ particles generated $\mathrm{ROS}$ at more than $125 \mu \mathrm{g} / \mathrm{mL}$ after $24 \mathrm{~h}$ 
(Figure 8A), regardless of particle size distribution (T3 or T4) or particle type (food- or general-grade). It is likely that the size is not a critical factor affecting the cytotoxicity of $\mathrm{TiO}_{2}$. Other reports demonstrated that $\mathrm{TiO}_{2}$ particles generated $\mathrm{ROS}$ but did not affect cell viability at similar concentrations used in this study [37-39]. High ROS generation but relatively low apoptosis induction by $\mathrm{TiO}_{2}$ particles were also reported [40-42], which is consistent with our results. Indeed, ROS generation and oxidative stress play roles in apoptosis induction $[43,44]$, but more exposure time or high concentration seems to be needed to examine apoptosis. $\mathrm{The}^{\mathrm{TiO}_{2}}$ particles were reported to cause oxidative stress [9], which may lead to DNA damage, inflammation, and possibly cancer [45-47]. Hence, the cytotoxicity potential of $\mathrm{TiO}_{2}$ is likely to be associated with its chemical characteristics, not with particle size. Further study is required to elucidate the toxicity mechanism and chronic toxicity potential of food-additive $\mathrm{TiO}_{2}$ particles.

On the other hand, the intestinal transport mechanism of $\mathrm{TiO}_{2}$ was determined to be by both $\mathrm{M}$ cells and Caco- 2 monolayer, and its primary transportation route was by $\mathrm{M}$ cells, showing extremely low transport amount (total 0.12-0.14\%) (Figure 9). The result obtained in in vitro 2D and 3D culture models was highly consistent with in vivo absorption after oral administration of food- or general-grade $\mathrm{TiO}_{2}$ particles to rats $[16,22]$. It is important to note that particle size distribution or particle type did not significantly affect the intestinal transport amount and mechanism of $\mathrm{TiO}_{2}$ particles.

\section{Conclusions}

In this study, commercially available food additive $\mathrm{TiO}_{2}$ particles were characterized and identified in commercial foods. The results obtained demonstrated that $\mathrm{TiO}_{2}$ was a material mainly consisting of larger than $100 \mathrm{~nm}$, and its fate in commercial foods was an agglomerated or aggregated particle, without significant decomposition in food matrices. Hence, $\mathrm{E} 171 \mathrm{TiO}_{2}$ is not a nanostructured material. Less than $1 \% \mathrm{TiO}_{2}$ were quantitatively detected in all commercial food products. The cytotoxicity of $\mathrm{TiO}_{2}$ was mainly associated with ROS generation and long-term inhibition of colony-forming ability only at high concentration, but particle size distribution or particle type (food- or general-grade) did not affect the cytotoxicity or intestinal transport amount and mechanism. These findings will be useful for $\mathrm{TiO}_{2}$ application in the food industry and predicting its potential toxicity.

Supplementary Materials: The following are available online at http://www.mdpi.com/2079-4991/9/8/1175/s1. Figure S1: X-ray diffraction (XRD) patterns of commercially available food additive $\mathrm{TiO}_{2}(\mathrm{~T} 1-\mathrm{T} 5)$ particles; Figure S2: Energy-dispersive X-ray spectroscopy (EDS) spectra of particles recovered from commercial foods; Table S1: Normal distribution fitting result for T4 before and after size fractionation using sucrose gradient solution.

Author Contributions: S.-J.C. conceived and designed the experiments, and wrote the paper; J.-S.H. performed physicochemical characterization and quantitative analysis and analyzed the data; J.Y. carried out cell experiments; H.-M.K. performed physicochemical characterization of pristine materials; J.-M.O. designed simulated food condition and analyzed physicochemical data.

Funding: This research was funded by a grant (18163MFDS011) from the Ministry of Food and Drug Safety 2018, by the National Research Foundation of Korea (NRF) grant funded by the Korea government (MSIT) (No. 2018R1A2B6001238), and by a research grant from Seoul Women's University (2019).

Conflicts of Interest: The authors declare no conflict of interest. The founding sponsors had no role in the design of the study; in the collection, analysis, or interpretation of data; in the writing of the manuscript, and in the decision to publish the results.

\section{References}

1. European Food Safety Authority (EFSA). Re-evaluation of titanium dioxide (E 171) as a food additive. EFSA J. 2016, 14, 1-83.

2. Dudefoi, W.; Moniz, K.; Allen-Vercoe, E.; Ropers, M.H.; Walker, V.K. Impact of food grade and nano-TiO 2 particles on a human intestinal community. Food Chem. Toxicol. 2017, 106, 242-249. [CrossRef] [PubMed]

3. Proquin, H.; Rodríguez-Ibarra, C.; Moonen, C.G.; Urrutia Ortega, I.M.; Briedé, J.J.; de Kok, T.M.; van Loveren, H.; Chirino, Y.I. Titanium dioxide food additive (E171) induces ROS formation and genotoxicity: Contribution of micro and nano-sized fractions. Mutagenesis 2017, 32, 139-149. [CrossRef] [PubMed] 
4. Heringa, M.B.; Geraets, L.; van Eijkeren, J.C.; Vandebriel, R.J.; de Jong, W.H.; Oomen, A.G. Risk assessment of titanium dioxide nanoparticles via oral exposure, including toxicokinetic considerations. Nanotoxicology 2016, 10, 1515-1525. [CrossRef] [PubMed]

5. Winkler, H.C.; Notter, T.; Meyer, U.; Naegeli, H. Critical review of the safety assessment of titanium dioxide additives in food. J. Nanobiotechnol. 2018, 16, 1-19. [CrossRef] [PubMed]

6. Kamat, P.V. Dominance of metal oxides in the era of nanotechnology. J. Phys. Chem. Lett. 2011, 2, 839-840. [CrossRef]

7. U.S. Food and Drug Administration. Available online: https://www.accessdata.fda.gov/scripts/cdrh/cfdocs/ cfCFR/CFRSearch.cfm?fr=73.575 (accessed on 25 July 2019).

8. European Commission. Commission Recommendation of 18 October 2011 on the definition of nanomaterial. Off. J. Eur. Union 2011, 275, 38-40.

9. Yang, Y.; Doudrick, K.; Bi, X.; Hristovski, K.; Herckes, P.; Westerhoff, P.; Kaegi, R. Characterization of food-grade titanium dioxide: The presence of nanosized particles. Environ. Sci. Technol. 2014, 48, 6391-6400. [CrossRef]

10. Weir, A.; Westerhoff, P.; Fabricius, L.; Hristovski, K.; von Goetz, N. Titanium dioxide nanoparticles in food and personal care products. Environ. Sci. Technol. 2012, 46, 2242-2250. [CrossRef]

11. Peters, R.J.B.; van Bemmel, G.; Herrera-Rivera, Z.; Helsper, H.P.F.G.; Marvin, H.J.P.; Weigel, S.; Tromp, P.C.; Oomen, A.G.; Rietveld, A.G.; Bouwmeester, H. Characterization of titanium dioxide nanoparticles in food products: Analytical methods to define nanoparticles. J. Agric. Food. Chem. 2014, 62, 6285-6293. [CrossRef]

12. Candás-Zapico, S.; Kutscher, D.J.; Montes-Bayón, M.; Bettmer, J. Single particle analysis of $\mathrm{TiO}_{2}$ in candy products using triple quadrupole ICP-MS. Talanta 2018, 180, 309-315. [CrossRef] [PubMed]

13. Singh, G.; Stephan, C.; Westerhoff, P.; Carlander, D.; Duncan, T.V. Measurement methods to detect, characterize, and quantify engineered nanomaterials in foods. Compr. Rev. Food Sci. F. 2014, 13, 693-704. [CrossRef]

14. Contado, C. Nanomaterials in consumer products: A challenging analytical problem. Front. Chem. 2015, 3, 48. [CrossRef] [PubMed]

15. Martirosyan, A.; Schneider, Y.J. Engineered nanomaterials in food: Implications for food safety and consumer health. Int. J. Env. Res. Public Health 2014, 11, 5720-5750. [CrossRef]

16. Jo, M.R.; Yu, J.; Kim, H.J.; Song, J.H.; Kim, K.M.; Oh, J.M.; Choi, S.J. Titanium dioxide nanoparticle-biomolecule interactions influence oral absorption. Nanomaterials 2016, 6, 225. [CrossRef]

17. Go, M.R.; Bae, S.H.; Kim, H.J.; Yu, J.; Choi, S.J. Interactions between food additive silica nanoparticles and food matrices. Front. Microbiol. 2017, 8, 1013. [CrossRef]

18. Lee, J.A.; Kim, M.K.; Song, J.H.; Jo, M.R.; Yu, J.; Kim, K.M.; Kim, Y.R.; Oh, J.M.; Choi, S.J. Biokinetics of food additive silica nanoparticles and their interactions with food components. Colloids Surf. B 2017, 150, 384-392. [CrossRef]

19. Cho, W.S.; Kang, B.C.; Lee, J.K.; Jeong, J.; Che, J.H.; Seok, S.H. Comparative absorption, distribution, and excretion of titanium dioxide and zinc oxide nanoparticles after repeated oral administration. Part. Fibre Toxicol. 2013, 10, 9. [CrossRef]

20. Geraets, L.; Oomen, A.G.; Krystek, P.; Jacobsen, N.R.; Wallin, H.; Laurentie, M.; Verharen, H.W.; Brandon, E.F.A.; de Jong, W.H. Tissue distribution and elimination after oral and intravenous administration of different titanium dioxide nanoparticles in rats. Part. Fibre Toxicol. 2014, 11, 30. [CrossRef]

21. Janer, G.; Mas del Molino, E.; Fernández-Rosas, E.; Fernández, A.; Vázquez-Campos, S. Cell uptake and oral absorption of titanium dioxide nanoparticles. Toxicol. Lett. 2014, 228, 103-110. [CrossRef]

22. Kim, M.K.; Lee, J.A.; Jo, M.R.; Choi, S.J. Bioavailability of silica, titanium dioxide, and zinc oxide nanoparticles in rats. J. Nanosci. Nanotechnol. 2016, 16, 6580-6586. [CrossRef] [PubMed]

23. Faust, J.J.; Doudrick, K.; Yang, Y.; Westerhoff, P.; Capco, D.G. Food grade titanium dioxide disrupts intestinal brush border microvilli in vitro independent of sedimentation. Cell Biol. Toxicol. 2014, 30, 169-188. [CrossRef] [PubMed]

24. Bettini, S.; Boutet-Robinet, E.; Cartier, C.; Coméra, C.; Gaultier, E.; Dupuy, J.; Naud, N.; Taché, S.; Grysan, P.; Reguer, $\mathrm{S}$; ; et al. Food-grade $\mathrm{TiO}_{2}$ impairs intestinal and systemic immune homeostasis, initiates preneoplastic lesions and promotes aberrant crypt development in the rat colon. Sci. Rep. 2017, 7, 40373. [CrossRef] [PubMed] 
25. Zhang, L.; Xie, X.; Zhou, Y.; Yu, D.; Deng, Y.; Ouyang, J.; Yang, B.; Luo, D.; Zhang, D.; Kuang, H. Gestational exposure to titanium dioxide nanoparticles impairs the placentation through dysregulation of vascularization, proliferation and apoptosis in mice. Int. J. Nanomed. 2018, 13, 777-789. [CrossRef] [PubMed]

26. Dorier, M.; Béal, D.; Tisseyre, C.; Marie-Desvergne, C.; Dubosson, M.; Barreau, F.; Houdeau, E.; Herlin-Boime, N.; Rabilloud, T.; Carriere, M. The food additive E171 and titanium dioxide nanoparticles indirectly alter the homeostasis of human intestinal epithelial cells in vitro. Environ. Sci. Nano 2019, 6, 1549-1561. [CrossRef]

27. Kim, N.; Kim, C.; Jung, S.; Park, Y.; Lee, Y.; Jo, J.; Hong, M.; Lee, S.; Oh, Y.; Jung, K. Determination and identification of titanium dioxide nanoparticles in confectionery foods, marketed in South Korea, using inductively coupled plasma optical emission spectrometry and transmission electron microscopy. Food Addit. Contam. A 2018, 35, 1238-1246. [CrossRef] [PubMed]

28. des Rieux, A.; Fievez, V.; Théate, I.; Mast, J.; Préat, V.; Schneider, Y.J. An improved in vitro model of human intestinal follicle-associated epithelium to study nanoparticle transport by M cells. Eur. J. Pharm. Sci. 2007, 30, 380-391. [CrossRef] [PubMed]

29. Fenton, H.J.H. Notes on Qualitative Anlaysis, Concise and Explanatory; Cambridge University Press: Cambridge, UK, 1923.

30. Walker, P.; Tarn, W.H. CRC Handbook of Metal Etchants; CRC Press: Boca Raton, FL, USA, 1991.

31. Korn, M.G.A.; Morte, E.S.B.; dos Santos, D.C.M.B.; Castro, J.T.; Barbosa, J.T.P.; Teixeira, A.P.; Fernandes, A.P.; Welz, B.; dos Santos, W.P.C.; dos Santos, E.B.G.N.; et al. Sample preparation for the determination of metals in food samples using spectroanalytical methods-A review. Appl. Spectrosc. Rev. 2008, 43, 67-92. [CrossRef]

32. Nardi, E.P.; Evangelista, F.S.; Tormen, L.; Saint’’Pierre, T.D.; Curtius, A.J.; de Souza, S.S.; Barbosa, F., Jr. The use of inductively coupled plasma mass spectrometry (ICP-MS) for the determination of toxic and essential elements in different types of food samples. Food Chem. 2009, 112, 727-732. [CrossRef]

33. Ito, S.; Kitamura, T.; Wada, Y.; Yanagida, S. Facile fabrication of mesoporous $\mathrm{TiO}_{2}$ electrodes for dye solar cells: Chemical modification and repetitive coating. Sol. Energy Mater. Sol. Cells 2003, 76, 3-13. [CrossRef]

34. Park, K.H.; Jin, E.M.; Gu, H.B.; Shim, S.E.; Hong, C.K. Effects of $\mathrm{HNO}_{3}$ treatment of $\mathrm{TiO}_{2}$ nanoparticles on the photovoltaic properties of dye-sensitized solar cells. Mater. Lett. 2009, 63, 2208-2211. [CrossRef]

35. Bunhu, T.; Kindness, A.; Martincigh, B.S. Determination of titanium dioxide in commercial sunscreens by inductively coupled plasma-optical emission spectrometry. S. Afr. J. Chem. 2011, 64, 139-143.

36. Dos Santos, E.J.; Dos Santos, M.P.; Herrmann, A.B.; Sturgeon, R.E. Rapid determination of Ti in $\mathrm{TiO}_{2}$ by $\mathrm{ICP}$ OES. Anal. Methods-UK 2016, 8, 6463-6467. [CrossRef]

37. Ekstrand-Hammarström, B.; Akfur, C.M.; Andersson, P.O.; Lejon, C.; Österlund, L.; Bucht, A. Human primary bronchial epithelial cells respond differently to titanium dioxide nanoparticles than the lung epithelial cell lines A549 and BEAS-2B. Nanotoxicology 2012, 6, 623-634. [CrossRef] [PubMed]

38. Dorier, M.; Brun, E.; Veronesi, G.; Barreau, F.; Pernet-Gallay, K.; Desvergne, C.; Rabilloud, T.; Carapito, C.; Herlin-Boime, N.; Carrière, M. Impact of anatase and rutile titanium dioxide nanoparticles on uptake carriers and efflux pumps in Caco-2 gut epithelial cells. Nanoscale 2015, 11, 7352-7360. [CrossRef] [PubMed]

39. Dorier, M.; Béal, D.; Marie-Desvergne, C.; Dubosson, M.; Barreau, F.; Houdeau, E.; Herlin-Boime, N.; Carriere, M. Continuous in vitro exposure of intestinal epithelial cells to E171 food additive causes oxidative stress, inducing oxidation of DNA bases but no endoplasmic reticulum stress. Nanotoxicology 2017, 11,751-761. [CrossRef] [PubMed]

40. Liu, S.; Xu, L.; Zhang, T.; Ren, G.; Yang, Z. Oxidative stress and apoptosis induced by nanosized titanium dioxide in PC12 cells. Toxicology 2010, 267, 172-177. [CrossRef] [PubMed]

41. Shi, Y.; Wang, F.; He, J.; Yadav, S.; Wang, H. Titanium dioxide nanoparticles cause apoptosis in BEAS-2B cells through the caspase 8/t-Bid-independent mitochondrial pathway. Toxicol. Lett. 2010, 196, 21-27. [CrossRef] [PubMed]

42. Srivastava, R.K.; Rahman, Q.; Kashyap, M.P.; Singh, A.K.; Jain, G.; Jahan, S.; Lohani, M.; Lantow, M.; Pant, A.B. Nano-titanium dioxide induces genotoxicity and apoptosis in human lung cancer cell line, A549. Hum. Exp. Toxicol. 2013, 32, 153-166. [CrossRef]

43. Circu, M.L.; Aw, T.Y. Reactive oxygen species, cellular redox systems, and apoptosis. Free Radic. Biol. Med. 2010, 48, 749-762. [CrossRef]

44. Redza-Dutordoir, M.; Averill-Bates, D.A. Activation of apoptosis signalling pathways by reactive oxygen species. Biochim. Biophys. Acta 2016, 1863, 2977-2992. [CrossRef] [PubMed] 
45. Bhattacharya, K.; Davoren, M.; Boertz, J.; Schins, R.P.; Hoffmann, E.; Dopp, E. Titanium dioxide nanoparticles induce oxidative stress and DNA-adduct formation but not DNA-breakage in human lung cells. Part. Fibre Toxicol. 2009, 6, 17. [CrossRef] [PubMed]

46. Trouiller, B.; Reliene, R.; Westbrook, A.; Solaimani, P.; Schiestl, R.H. Titanium dioxide nanoparticles induce DNA damage and genetic instability in vivo in mice. Cancer Res. 2009, 69, 8784-8789. [CrossRef] [PubMed]

47. Han, S.G.; Newsome, B.; Hennig, B. Titanium dioxide nanoparticles increase inflammatory responses in vascular endothelial cells. Toxicology 2013, 306, 1-8. [CrossRef] [PubMed]

(C) 2019 by the authors. Licensee MDPI, Basel, Switzerland. This article is an open access article distributed under the terms and conditions of the Creative Commons Attribution (CC BY) license (http://creativecommons.org/licenses/by/4.0/). 